\title{
A Climate Event Portal co-created by Citizen Scientists
}

\author{
Andreas Divanis ${ }^{\mathrm{a}}, *$, Liqiu Meng ${ }^{\text {a }}$ \\ ${ }^{a}$ Chair of Cartography, Technical University of Munich, 80333 Munich, Germany, andreas.divanis@tum.de, liqiu.meng@tum.de \\ * Corresponding author
}

Keywords: Citizen Science, Participatory Science, Web Portal, Open Science

\begin{abstract}
:
Citizen Science projects rely on the public participation of various forms, such as in data collection, classification, or more advanced analytical processes. If we allow the public involvement to be more than just being partners for data collection and classification, we are moving towards the so-called "extreme citizen science". Such practice takes into account local needs and works together with public users to build features and design tools with the aim to meet the needs of these public users and scientists. Following the approach of "extreme citizen science", we are building a Citizen Science portal to explore what kind of features, or changes are desirable from the citizens' perspective.
\end{abstract}

The portal is called BAYSICS (Bavarian Synthesis Information Citizen Science Portal for Climate Research and Science Communication) funded by the Bavarian State Ministry of Science and Art. It has the objective to tackle the misconception that climate change is happening far away from our doorsteps and to communicate scientific processes in a more transparent way to citizens by involving them. While the portal is open to the public, we also have secured cooperation with 55 schools in Bavaria that will use its features in teaching. On the one hand, our users may want certain functionality related to policy-making, such as visualization at the municipal administrative level, on the other hand, they may find features to visualize certain data or phenomena useful as a teaching tool.

The data collected in the portal and the mobile application fall into four different categories (1) the yearly phenological development stages of plants sensitive to temperature; (2) trees in the alpine mountain ranges of Bavaria and comparing their elevation to the elevation of historical records; (3) allergenic species since pollen drift is sensitive to atmospheric conditions; and (4) animal observations in urban areas serving as testing sites to different conditions the non-urban areas. All the data have a temporal and spatial component and category-specific attributes. They are stored in a relational database (PostGIS), which is then displayed as a web map on our portal. Furthermore, all the collected data are open and free to download.

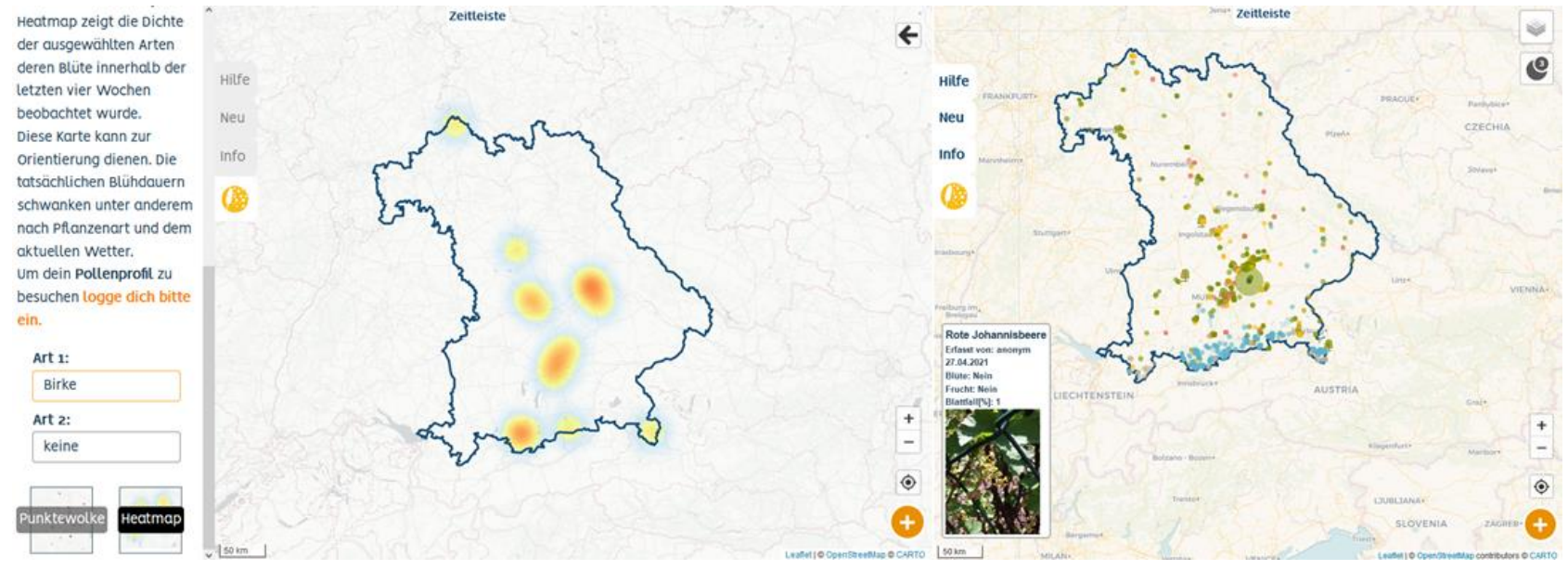

Figure 1. Map view of the portal

To collect desirable features for participants and to involve them in the designing process of the web portal we adopted a proactive approach. We regularly asked for feedback, we applied guerrilla testing by approaching people who have not 
been involved in the project yet and noted their ideas and difficulties, and on the portal and the website landing page, we emphasized that we are still in a designing stage and invited the users to send us suggestions for their desired features.

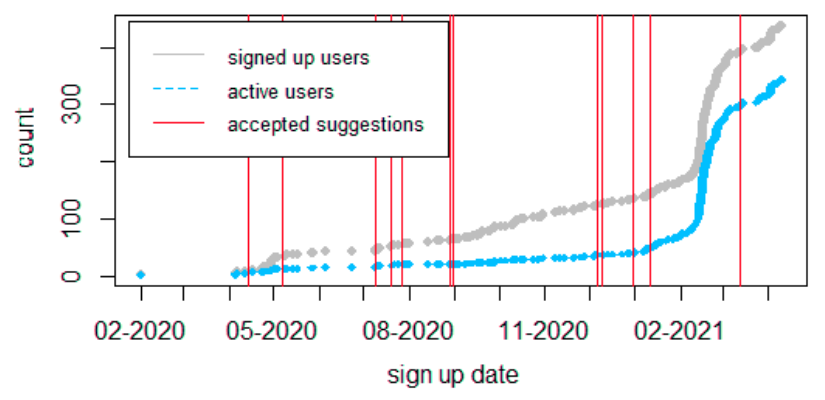

Figure 2. Signed up portal users, active users and accepted suggestions over time

As of May 2021, we had 438 users, 344 of which we considered as active based on their last login date (not older than 100 days). We accepted 36 user suggestions out of 48 since making the portal public in May 2020.

A majority of the requested features (Table 1) were related to the map view on our portal. Some features, however, were not feasible to implement, such as AI-help for automatic classification of certain observation types with a mobile phone camera, or if user suggestions clashed (e.g. smaller icons versus larger icons).

\begin{tabular}{|l|l|l|}
\hline User suggestions & implemented & $\begin{array}{l}\text { Not } \\
\text { implemented }\end{array}$ \\
\hline Change to existing map & 12 & 5 \\
New map-related feature & 10 & 1 \\
Not map related & 14 & 6 \\
\hline
\end{tabular}

Table 1. User-requested Changes

Desirable features for users included more background data regarding municipalities, topological maps, information to plan routes for collecting new data while hiking in mountain areas, as well as accuracy-feedback on determining the current GPS location when using the mobile application. User suggestions that aligned most with our agenda of involving the citizens beyond data collection, included requests for spatial visualization tools, such as an allergenic species risk map, which allows users to select certain species, compare the flowering period, and approximate the pollen drift.

Even though our data contain many more attributes than spatial ones, our results so far suggest that map-related features were the most frequently requested compared to other suggestions such as changes to a tabular view or statistical plots. Currently, the portal is released and operational, but is still in the construction phase and features are still being added. We expect an increase in active users after the pandemic, possibly at the start of the hiking season in the summer of 2021. This may lead to more and different desirable features suggested by users. 